\title{
GAIA Preterm Birth Level of Diagnostic Certainty
}

National Cancer Institute

\section{Source}

National Cancer Institute. GAIA Preterm Birth Level of Diagnostic Certainty. NCI

Thesaurus. Code C126845.

A classification of maternal and fetal outcomes relating to preterm birth, developed by the Global Alignment of Immunization safety Assessment in pregnancy, based on the extent to which the diagnosis has been confirmed. 